\title{
A nonmonotone hybrid conjugate gradient method for unconstrained optimization
}

Wenyu Li and Yueting Yang*

"Correspondence:

yangyueting@163.com

School of Mathematics and

Statistics, Beihua University, Jilin

Street No. 15, Jilin, China

\begin{abstract}
A nonmonotone hybrid conjugate gradient method is proposed, in which the technique of the nonmonotone Wolfe line search is used. Under mild assumptions, we prove the global convergence and linear convergence rate of the method. Numerical experiments are reported.
\end{abstract}

Keywords: unconstrained optimization; nonmonotone hybrid conjugate gradient algorithm; global convergence; linear convergence rate

\section{Introduction}

Let us take the following unconstrained optimization problem:

$$
\min _{x \in R^{n}} f(x)
$$

where $f: R^{n} \rightarrow R$ is continuously differentiable. For solving (1), the conjugate gradient method generates a sequence $\left\{x_{k}\right\}: x_{k+1}=x_{k}+\alpha_{k} d_{k}, d_{0}=-g_{0}$, and $d_{k}=-g_{k}+\beta_{k} d_{k-1}$, where the stepsize $\alpha_{k}>0$ is obtained by the line search, $d_{k}$ is the search direction, $g_{k}=\nabla f\left(x_{k}\right)$ is the gradient of $f(x)$ at the point $x_{k}$, and $\beta_{k}$ is known as the conjugate gradient parameter. Different parameters correspond to different conjugate gradient methods. A remarkable survey of conjugate gradient methods is given by Hager and Zhang [1].

Plenty of hybrid conjugate gradient methods were presented in [2-7] after the first hybrid conjugate algorithm was proposed by Touati-Ahmed and Storey [8]. In [5], Lu et al. proposed a new hybrid conjugate gradient method (LY) with the conjugate gradient parameter $\beta_{k}^{L Y}$,

$$
\beta_{k}^{L Y}= \begin{cases}\frac{g_{k}^{T}\left(g_{k}-d_{k-1}\right)}{d_{k-1}^{T}\left(g_{k}-g_{k-1}\right)}, & \text { if }\left|1-\frac{g_{k}^{T} d_{k-1}}{\left\|g_{k}\right\|^{2}}\right| \leq \mu, \\ \frac{\mu\left\|g_{k}\right\|^{2}}{d_{k-1}^{T} g_{k}-\lambda d_{k-1}^{T} g_{k-1}}, & \text { otherwise, }\end{cases}
$$

where $0<\mu \leq \frac{\lambda-\sigma}{1-\sigma}, \sigma<\lambda \leq 1$. Numerical experiments show that the LY method is effective.

It is well known that the nonmonotone algorithms are promising methods for solving highly nonlinear large-scale and possibly ill-conditioned problems. The first nonmonotone line search framework was proposed by Grippo et al. in [9] for Newton's methods.

\section{然 Springer}

(c) $2015 \mathrm{Li}$ and Yang; licensee Springer. This is an Open Access article distributed under the terms of the Creative Commons Attribution License (http://creativecommons.org/licenses/by/4.0), which permits unrestricted use, distribution, and reproduction in any medium, provided the original work is properly credited. 
At each iteration, the current function value is defined as follows:

$$
f_{l(k)}=\max _{0 \leq j \leq m(k)} f\left(x_{k-j}\right)
$$

where $m(0)=0,0 \leq m(k) \leq \min \{m(k-1)+1, M\}, M$ is some positive integer. Zhang and Hager [10] proposed another nonmonotone line search technique, they adopted $C_{k}$ to replace the current function $f_{k}$, where

$$
\begin{gathered}
C_{k}=\frac{\zeta_{k-1} Q_{k-1} C_{k-1}+f_{k}}{Q_{k}}, \\
Q_{0}=1, C_{0}=f\left(x_{0}\right), \zeta_{k-1} \in[0,1], \text { and } \\
Q_{k}=\zeta_{k-1} Q_{k-1}+1 .
\end{gathered}
$$

To obtain the global convergence (see $[4,11-14])$ and implement the algorithms, the line search in the conjugate gradient is usually chosen by a Wolfe line search; the stepsize $\alpha_{k}$ satisfies the following two inequalities:

$$
\begin{aligned}
& f\left(x_{k}+\alpha_{k} d_{k}\right) \leq f\left(x_{k}\right)+\rho \alpha_{k} g_{k}^{T} d_{k}, \\
& g\left(x_{k}+\alpha_{k} d_{k}\right)^{T} d_{k} \geq \sigma g_{k}^{T} d_{k},
\end{aligned}
$$

where $0<\rho<\sigma<1$. In particular, a nonmonotone version line search can relax the choice of the stepsize. Therefore the nonmonotone Wolfe line search requires the stepsize $\alpha_{k}$ to satisfy

$$
f\left(x_{k}+\alpha_{k} d_{k}\right) \leq f_{l(k)}+\rho \alpha_{k} g_{k}^{T} d_{k}
$$

and (7), or

$$
f\left(x_{k}+\alpha_{k} d_{k}\right) \leq C_{k}+\rho \alpha_{k} g_{k}^{T} d_{k}
$$

and (7).

The aim of this paper is to propose a nonmonotone hybrid conjugate gradient method which combines the nonmonotone line search technique with the LY method. It is based on the idea that the larger values of the stepsize $\alpha_{k}$ may be accepted by the nonmonotone algorithmic framework and improve the behavior of the LY method.

The paper is organized as follows. A new nonmonotone hybrid conjugate gradient algorithm is presented and the global convergence of the algorithm is proved in Section 2. The line convergence rate of the algorithm is shown in Section 3. In Section 4, numerical results are reported.

\section{Nonmonotone hybrid conjugate gradient algorithm and global convergence}

Now we present a nonmonotone hybrid conjugate gradient algorithm.

\section{Algorithm 1}

Step 1. Given $x_{0} \in R^{n}, \epsilon>0, d_{0}=-g_{0}, C_{0}=f_{0}, Q_{0}, \zeta_{0}, k:=0$. 
Step 2. If $\left\|g_{k}\right\|<\epsilon$, then stop. Otherwise, compute $\alpha_{k}$ by (9) and (7), set $x_{k+1}=x_{k}+\alpha_{k} d_{k}$.

Step 3. Compute $\beta_{k+1}$ by (2), set $d_{k+1}=-g_{k+1}+\beta_{k+1} d_{k}, k:=k+1$, and go to Step 2 .

Assumption 1 We make the following assumptions:

(i) The level set $\Omega_{0}=\left\{x \in R^{n}: f(x) \leq f\left(x_{0}\right)\right\}$ is bounded, where $x_{0}$ is the initial point.

(ii) The gradient function $g(x)=\nabla f(x)$ of the objective function $f$ is Lipschitz continuous in a neighborhood $\mathcal{N}$ of level set $\Omega_{0}$, i.e. there exists a constant $L \geq 0$ such that

$$
\|g(x)-g(\bar{x})\| \leq L\|x-\bar{x}\|,
$$

for any $x, \bar{x} \in \mathcal{N}$.

Lemma 2.1 Let the sequence $\left\{x_{k}\right\}$ be generated by Algorithm 1 . Then $d_{k}^{T} g_{k}<0$ holds for all $k \geq 1$.

Proof From Lemma 2 and Lemma 3 in [5], the conclusion holds.

Lemma 2.2 Let Assumption 1 hold and the sequence $\left\{x_{k}\right\}$ be obtained by Algorithm $1, \alpha_{k}$ satisfies the nonmonotone Wolfe conditions (9) and (7). Then

$$
\alpha_{k} \geq \frac{\sigma-1}{L} \frac{g_{k}^{T} d_{k}}{\left\|d_{k}\right\|^{2}}
$$

Proof From (7), we have

$$
\left(g_{k+1}-g_{k}\right)^{T} d_{k} \geq(\sigma-1) g_{k}^{T} d_{k}
$$

and by (ii) of Assumption 1 it implies that

$$
\left(g_{k+1}-g_{k}\right)^{T} d_{k} \leq \alpha_{k} L\left\|d_{k}\right\|^{2} .
$$

By combining these two inequalities, we obtain

$$
\alpha_{k} \geq \frac{\sigma-1}{L} \frac{g_{k}^{T} d_{k}}{\left\|d_{k}\right\|^{2}}
$$

Lemma 2.3 Let the sequence $\left\{x_{k}\right\}$ be generated by Algorithm 1 and $d_{k}^{T} g_{k}<0$ hold for all $k \geq 1$. Then

$$
f_{k} \leq C_{k}
$$

Proof See Lemma 1.1 in [10].

Lemma 2.4 Let Assumption 1 hold, and the sequence $\left\{x_{k}\right\}$ be obtained by Algorithm 1, where $d_{k}$ satisfies $d_{k}^{T} g_{k}<0, \alpha_{k}$ is obtained by the nonmonotone Wolfe conditions (9) and (7). 
Then

$$
\sum_{k \geq 0} \frac{1}{Q_{k+1}} \frac{\left(d_{k}^{T} g_{k}\right)^{2}}{\left\|d_{k}\right\|^{2}}<+\infty
$$

Proof By (9) and (10), we have

$$
f_{k+1} \leq C_{k}-c_{0} \frac{\left(d_{k}^{T} g_{k}\right)^{2}}{\left\|d_{k}\right\|^{2}}
$$

where $c_{0}=\rho(1-\sigma) / L$.

From (4), (5), and (13), we have

$$
C_{k+1}=\frac{\zeta_{k} Q_{k} C_{k}+f\left(x_{k+1}\right)}{Q_{k+1}} \leq \frac{\zeta_{k} Q_{k} C_{k}+C_{k}-c_{0} \frac{\left(d_{k}^{T} g_{k}\right)^{2}}{\left\|d_{k}\right\|^{2}}}{Q_{k+1}} \leq C_{k}-\frac{c_{0}}{Q_{k+1}} \frac{\left(d_{k}^{T} g_{k}\right)^{2}}{\left\|d_{k}\right\|^{2}} .
$$

Since $f(x)$ is bounded from below in the level set $\Omega_{0}$ and by (11) for all $k$, we know that $C_{k}$ is bounded from below. It follows from (14) that (12) holds.

Theorem 2.1 Suppose that Assumptions 1 hold and the sequence $\left\{x_{k}\right\}$ is generated by the Algorithm 1. If $\zeta_{\max }<1$, then either $g_{k}=0$ for some $k$ or

$$
\lim _{k \rightarrow \infty} \inf \left\|g_{k}\right\|=0 .
$$

Proof We prove by contradiction and assume that there exists a constant $\epsilon>0$ such that

$$
\left\|g_{k}\right\|^{2} \geq \epsilon, \quad k=0,1,2,3, \ldots
$$

By Lemma 4 in [5], we have $\left|\beta_{k}\right|^{L Y} \leq \frac{\mu\left\|g_{k}\right\|^{2}}{d_{k-1}^{T} g_{k}-\lambda d_{k-1}^{T} g_{k-1}}$. Then we have $\left\|d_{k}\right\|^{2}=\left(\beta^{L Y}\right)^{2} \times$ $\left\|d_{k-1}\right\|^{2}-2 g_{k}^{T} d_{k}-\left\|g_{k}\right\|^{2} \leq\left(\frac{\mu\left\|g_{k}\right\|^{2}}{d_{k-1}^{T} g_{k}-\lambda d_{k-1}^{T} g_{k-1}}\right)^{2}\left\|d_{k-1}\right\|^{2}-2 g_{k}^{T} d_{k}-\left\|g_{k}\right\|^{2}$. The rest of the proof is similar to Theorem 2 and Theorem 1 in [5], and we also conclude

$$
\frac{\left(g_{k}^{T} d_{k}\right)^{2}}{\left\|d_{k}\right\|^{2}} \geq \frac{\epsilon}{k}
$$

Furthermore, by $\zeta_{\max }<1$ and (5), we have

$$
Q_{k}=1+\sum_{j=0}^{k-1} \prod_{i=0}^{j} \zeta_{k-1-i} \leq \frac{1}{1-\zeta_{\max }},
$$

then

$$
\frac{1}{Q_{k+1}} \frac{\left(g_{k}^{T} d_{k}\right)^{2}}{\left\|d_{k}\right\|^{2}} \geq\left(1-\zeta_{\max }\right) \frac{\epsilon}{k},
$$

which indicates

$$
\sum_{i=1}^{\infty} \frac{1}{Q_{k+1}} \frac{\left(g_{k}^{T} d_{k}\right)^{2}}{\left\|d_{k}\right\|^{2}}=+\infty
$$

This contradicts (12). Therefore (15) holds. 


\section{Linear convergence rate of algorithm}

We analyze the linearly convergence rate of the nonmonotone hybrid conjugate gradient method under the uniform convex assumption of $f(x)$. The nonmonotone strong Wolfe line search is adopted in this section, given by (9) and

$$
\left|g\left(x_{k}+\alpha_{k} d_{k}\right)^{T} d_{k}\right| \leq-\sigma g_{k}^{T} d_{k}
$$

We suppose that the object function $f(x)$ is twice continuously differentiable and uniformly convex on the level set $\Omega_{0}$. Then the point $x^{*}$ denotes a unique solution of the problem (1); there exists a positive constant $\tau$ such that

$$
f(x)-f\left(x^{*}\right) \leq\|\nabla f(x)\|\left\|x-x^{*}\right\| \leq \tau\|\nabla f(x)\|^{2}, \quad \text { for all } x \in R^{n} .
$$

The above conclusion (19) can be found in [10].

To analyze the convergence of the nonmonotone line search hybrid conjugate gradient method, the main difficulty is that the search directions do not usually satisfy the direction condition:

$$
g_{k}^{T} d_{k} \leq-c\left\|g_{k}\right\|^{2}
$$

for some constant $c>0$ and all $k \geq 1$. The following lemma has proven that the direction generated by Algorithm 1 with the strong Wolfe line search (9) and (18) in this paper satisfies the direction condition (20) by the observation for $g_{k}^{T} d_{k-1}$.

Lemma 3.1 Suppose that the sequence $\left\{x_{k}\right\}$ is generated by Algorithm 1 with the strong Wolfe line search (9) and (18), $0<\sigma<\frac{\lambda}{1+\mu}$. Then there exists some constant $c>0$ such that the direction condition (20) holds.

Proof According to the choice of the conjugate gradient parameter $\beta_{k}^{L Y}$, the result is discussed by two cases. In the first case,

$$
\left|1-\frac{g_{k}^{T} d_{k-1}}{\left\|g_{k}\right\|^{2}}\right| \leq \mu, \quad \text { i.e. } 1-\mu \leq \frac{g_{k}^{T} d_{k-1}}{\left\|g_{k}\right\|^{2}} \leq 1+\mu,
$$

then $\beta_{k}=\frac{g_{k}^{T}\left(g_{k}-d_{k-1}\right)}{d_{k-1}^{T}\left(g_{k}-g_{k-1}\right)}$. If $\beta_{k} \geq 0$, then, by $(21)$ and $d_{k-1}^{T} g_{k-1}<0, d_{k-1}^{T} g_{k} \geq 0$ and $g_{k}^{T}\left(g_{k}-d_{k-1}\right) \geq$ 0 . Furthermore, we have, by (18),

$$
\begin{aligned}
g_{k}^{T} d_{k} & =-\left\|g_{k}\right\|^{2}+\frac{g_{k}^{T}\left(g_{k}-d_{k-1}\right)}{d_{k-1}^{T}\left(g_{k}-g_{k-1}\right)} g_{k}^{T} d_{k-1} \\
& \leq-\left\|g_{k}\right\|^{2}-\sigma \frac{g_{k}^{T}\left(g_{k}-d_{k-1}\right)}{d_{k-1}^{T}\left(g_{k}-g_{k-1}\right)} g_{k-1}^{T} d_{k-1} \\
& \leq-\left\|g_{k}\right\|^{2}-\sigma \frac{g_{k}^{T}\left(g_{k}-d_{k-1}\right)}{-d_{k-1}^{T} g_{k-1}} g_{k-1}^{T} d_{k-1} \\
& =-\left\|g_{k}\right\|^{2}+\sigma\left(\left\|g_{k}\right\|^{2}-g_{k}^{T} d_{k-1}\right) \\
& =-(1-\sigma)\left\|g_{k}\right\|^{2}-\sigma g_{k}^{T} d_{k-1}
\end{aligned}
$$




$$
\begin{aligned}
& \leq-(1-\sigma)\left\|g_{k}\right\|^{2}-\sigma(1-\mu)\left\|g_{k}\right\|^{2} \\
& =-(1-\sigma \mu)\left\|g_{k}\right\|^{2} .
\end{aligned}
$$

If $\beta_{k}<0$, we have, by (18) and (21),

$$
\begin{aligned}
g_{k}^{T} d_{k} & =-\left\|g_{k}\right\|^{2}+\frac{g_{k}^{T}\left(g_{k}-d_{k-1}\right)}{d_{k-1}^{T}\left(g_{k}-g_{k-1}\right)} g_{k}^{T} d_{k-1} \\
& \leq-\left\|g_{k}\right\|^{2}+\sigma \frac{g_{k}^{T}\left(g_{k}-d_{k-1}\right)}{d_{k-1}^{T}\left(g_{k}-g_{k-1}\right)} g_{k-1}^{T} d_{k-1} \\
& \leq-\left\|g_{k}\right\|^{2}+\sigma \frac{g_{k}^{T}\left(g_{k}-d_{k-1}\right)}{-d_{k-1}^{T} g_{k-1}} g_{k-1}^{T} d_{k-1} \\
& \leq-\left\|g_{k}\right\|^{2}-\sigma\left(\left\|g_{k}\right\|^{2}-g_{k}^{T} d_{k-1}\right) \\
& =-(1+\sigma)\left\|g_{k}\right\|^{2}+\sigma g_{k}^{T} d_{k-1} \\
& =-(1+\sigma)\left\|g_{k}\right\|^{2}+\sigma(1+\mu)\left\|g_{k}\right\|^{2} \\
& =-(1-\sigma \mu)\left\|g_{k}\right\|^{2} .
\end{aligned}
$$

In the second case,

$$
\left|1-\frac{g_{k}^{T} d_{k-1}}{\left\|g_{k}\right\|^{2}}\right|>\mu,
$$

then $\beta_{k}=\frac{\mu\left\|g_{k}\right\|^{2}}{d_{k-1}^{T} g_{k}-\lambda d_{k-1}^{T} g_{k-1}}>0$. By (18), we have

$$
\begin{aligned}
g_{k}^{T} d_{k} & =-\left\|g_{k}\right\|^{2}+\frac{\mu\left\|g_{k}\right\|^{2}}{d_{k-1}^{T} g_{k}-\lambda d_{k-1}^{T} g_{k-1}} g_{k}^{T} d_{k-1} \\
& \leq-\left\|g_{k}\right\|^{2}-\frac{\sigma \mu\left\|g_{k}\right\|^{2}}{(\sigma-\lambda) d_{k-1}^{T} g_{k-1}} g_{k-1}^{T} d_{k-1} \\
& =-\left(1-\frac{\mu \sigma}{\lambda-\sigma}\right)\left\|g_{k}\right\|^{2} .
\end{aligned}
$$

From (23) and (25), we obtain (20), where $c=\min \left\{1-\sigma \mu, 1-\frac{\mu \sigma}{\lambda-\sigma}\right\}>0$. The proof is completed.

Lemma 3.2 Suppose the assumptions of Lemma 2.2 hold and, for all $k$,

$$
\left\|d_{k}\right\| \leq c_{1}\left\|g_{k}\right\|
$$

then there exists a constant $c_{2}>0$ such that

$$
\alpha_{k} \geq c_{2}, \quad \text { for all } k \text {. }
$$

Proof By Lemma 2.2 and Lemma 3.2, we have

$$
\alpha_{k} \geq \frac{\sigma-1}{L} \frac{g_{k}^{T} d_{k}}{\left\|d_{k}\right\|^{2}} \geq-\frac{\sigma-1}{L} \frac{c\left\|g_{k}\right\|^{2}}{c_{1}\left\|g_{k}\right\|^{2}} \geq c_{2},
$$

where $c_{2}=\frac{c(1-\sigma)}{c_{1} L}$. 
Table 1 Test problems

\begin{aligned} & \hline No. Problem name \\ & \hline 1 Helical valley function \\ & 2 BIGGS6 \\ & 3 Gaussian function \\ & 4 POWELLBS \\ & 5 BOX3 \\ & 6 BEALE \\ & 7 WOODS \\ & 8 FREUROTH \\ & 9 Osborne 1 function \\ & 10 Osborne 2 function \\ & 11 Powell singular function \\ & 12 Meyer function \\ & 13 Bard function \\ & 14 PENALTY2 \\ & 15 VARDIM \\ & 16 PENALTY1 \\ & 17 EXTROSNB \\ & 18 EXtended Powell singular function \\ & 19 CHEBYQAD \\ & 20 BROYDN3D \\ & \hline\end{aligned}

\begin{tabular}{ll}
\hline No. & Problem name \\
\hline 21 & Separable cubic function \\
22 & Nearly separable function \\
23 & Allgower function \\
24 & Schittkowski function 302 \\
25 & Discrete integral equation function \\
26 & BDQRTIC \\
27 & ARGLINB \\
28 & ARWHEAD \\
29 & NONDIA \\
30 & NONDQUAR \\
31 & DQDRTIC \\
32 & EG2 \\
33 & CURLY20 \\
34 & LIARWHD \\
35 & ENGVAL1 \\
36 & CRAGGLVY \\
37 & the Edensch function \\
38 & the Explin 1 function \\
39 & the Argling function \\
40 & NONSCOMP \\
\hline
\end{tabular}

Theorem 3.1 Let $x^{*}$ be the unique solution of problem (1) and the sequence $\left\{x_{k}\right\}$ be generated by Algorithm 1 with the nonmonotone Wolfe conditions (9) and (18), $0<\sigma<\frac{\lambda}{1+\mu}$. If $\alpha_{k} \leq v$ and $\zeta_{\max }<1$, then there exists a constant $\vartheta \in(0,1)$ such that

$$
f_{k}-f\left(x^{*}\right) \leq \vartheta^{k}\left(f_{0}-f\left(x^{*}\right)\right)
$$

Proof The proof is similar to that Theorem 3.1 given in [10]. By (9), (20), and (27), we have

$$
f_{k+1} \leq C_{k}+\rho \alpha_{k} g_{k}^{T} d_{k} \leq C_{k}-c c_{2} \rho\left\|g_{k}\right\|^{2} .
$$

By (ii) in Assumption 1, $x_{k+1}=x_{k}+\alpha_{k} d_{k}$ and (27), we have

$$
\left\|g_{k+1}\right\| \leq\left\|g_{k+1}-g_{k}\right\|+\left\|g_{k}\right\| \leq \alpha_{k} L\left\|d_{k}\right\|+\left\|g_{k}\right\| \leq\left(1+c_{1} \nu L\right)\left\|g_{k}\right\| .
$$

In the first case, $\left\|g_{k}\right\|^{2} \geq \beta\left(C_{k}-f\left(x^{*}\right)\right)$, where

$$
\beta=1 /\left(c c_{2} \rho+\tau\left(1+c_{1} v L\right)^{2}\right) .
$$

By (4) and (29), we have

$$
\begin{aligned}
C_{k+1}-f\left(x^{*}\right) & =\frac{\zeta_{k} Q_{k}\left(C_{k}-f\left(x^{*}\right)\right)+\left(f_{k+1}-f\left(x^{*}\right)\right)}{1+\zeta_{k} Q_{k}} \\
& \leq \frac{\zeta_{k} Q_{k}\left(C_{k}-f\left(x^{*}\right)\right)+\left(C_{k}-f\left(x^{*}\right)\right)-c c_{2} \rho\left\|g_{k}\right\|^{2}}{1+\zeta_{k} Q_{k}} \\
& =C_{k}-f\left(x^{*}\right)-\frac{c c_{2} \rho\left\|g_{k}\right\|^{2}}{Q_{k+1}} .
\end{aligned}
$$

Since $Q_{k+1} \leq \frac{1}{1-\zeta_{\max }}$ by (17), we have

$$
C_{k+1}-f\left(x^{*}\right) \leq C_{k}-f\left(x^{*}\right)-c c_{2} \rho\left(1-\zeta_{\max }\right)\left\|g_{k}\right\|^{2} .
$$


Table 2 Numerical comparisons of LY, NMLY1, and NMLY2

\begin{tabular}{|c|c|c|c|c|c|c|c|c|c|c|c|c|c|}
\hline \multirow[t]{2}{*}{ No. } & \multirow[t]{2}{*}{ Dim } & \multicolumn{4}{|l|}{ LY } & \multicolumn{4}{|c|}{ NMLY2 } & \multicolumn{4}{|c|}{ NMLY1 } \\
\hline & & it & nf & $\mathrm{ng}$ & $t$ & it & nf & $\mathrm{ng}$ & $t$ & it & nf & $\mathrm{ng}$ & $t$ \\
\hline 1 & 3 & 36 & 69 & 48 & 0.0156 & 59 & 137 & 92 & 0.0156 & 40 & 84 & 60 & 0.0156 \\
\hline 2 & 6 & 147 & 243 & 200 & 0.0468 & 413 & 853 & 697 & 0.1404 & 146 & 270 & 213 & 0.0468 \\
\hline 3 & 3 & 2 & 5 & 4 & 0 & 4 & 10 & 8 & 0 & 3 & 9 & 8 & 0 \\
\hline 4 & 2 & 9 & 26 & 13 & 0 & 11 & 48 & 28 & 0 & 13 & 38 & 17 & 0.0312 \\
\hline 5 & 3 & 15 & 28 & 19 & 0 & 29 & 75 & 68 & 0.0312 & 14 & 35 & 28 & 0 \\
\hline 6 & 2 & 37 & 61 & 46 & 0.0156 & 28 & 64 & 45 & 0 & 20 & 60 & 30 & 0.0156 \\
\hline 7 & 4 & 74 & 130 & 100 & 0.0156 & 313 & 623 & 458 & 0.0936 & 62 & 119 & 90 & 0.0156 \\
\hline 8 & 2 & 44 & 82 & 61 & 0.0156 & 37 & 98 & 70 & 0 & 5 & 25 & 9 & 0.0156 \\
\hline 9 & 5 & 128 & 233 & 184 & 0.0936 & 181 & 383 & 278 & 0.1404 & 104 & 199 & 153 & 0.078 \\
\hline 10 & 11 & 3 & 42 & 3 & 0.01 & 4 & 29 & 4 & 0 & 3 & 13 & 3 & 0.0312 \\
\hline 11 & 4 & 6 & 49 & 7 & 0 & 169 & 348 & 254 & 0.0468 & 59 & 121 & 77 & 0.0312 \\
\hline 12 & 3 & 9 & 45 & 14 & 0 & 170 & 456 & 328 & 0.12 & 13 & 80 & 34 & 0.0156 \\
\hline 13 & 3 & 25 & 49 & 33 & 0.0156 & 64 & 135 & 101 & & 39 & 75 & 52 & \\
\hline 14 & 500 & 14 & 70 & 28 & 0.01 & 10 & 82 & 40 & 0.0 & 3 & 46 & 10 & 0.0156 \\
\hline \multirow[t]{3}{*}{15} & 500 & 13 & 68 & 14 & 0 & 13 & 118 & 48 & 0.0312 & 17 & 187 & 122 & 0.0156 \\
\hline & 1,000 & 16 & 70 & 16 & 0 & 13 & 87 & 35 & 0.0 & 19 & 301 & 211 & 0.0312 \\
\hline & 2,000 & 14 & 58 & 14 & 0.0156 & 15 & 116 & 50 & 0.0 & 26 & 362 & 237 & 0.0624 \\
\hline \multirow[t]{4}{*}{16} & 500 & 11 & 35 & 22 & 0.015 & 40 & 147 & 101 & & 34 & 103 & 87 & 0.0156 \\
\hline & 1,000 & 16 & 40 & 23 & 0 & 49 & 141 & 99 & & 21 & 82 & 62 & 56 \\
\hline & 2,000 & 20 & 51 & 28 & 0.03 & 34 & 122 & 73 & & 57 & 156 & 108 & \\
\hline & 1,000 & 22 & 75 & 30 & & 28 & 96 & 67 & & 31 & 134 & 70 & 0.1 \\
\hline \multirow[t]{4}{*}{17} & 500 & 42 & 87 & 58 & 0.01 & 133 & 294 & 213 & & 3 & 18 & 3 & 0 \\
\hline & 1,000 & 64 & 120 & 87 & 0.03 & 187 & 406 & 299 & & 3 & 19 & 3 & 0 \\
\hline & 2,000 & 53 & 105 & 72 & 0.03 & 134 & 304 & 221 & & 3 & 19 & 3 & 0.0156 \\
\hline & 1,000 & 56 & 113 & 79 & 0.20 & 184 & 393 & 292 & & 3 & 19 & 3 & \\
\hline \multirow[t]{4}{*}{18} & 500 & 220 & 364 & 295 & 0.10 & 184 & 353 & 253 & & 110 & 197 & 160 & 0.0624 \\
\hline & 1,000 & 180 & 288 & 238 & 0.14 & 163 & 322 & 235 & & 104 & 191 & 150 & 0.078 \\
\hline & 2,000 & 181 & 308 & 248 & & 181 & 361 & 262 & & 112 & 205 & 164 & \\
\hline & 1,000 & 21 & 361 & 293 & & 320 & & & & 133 & & 197 & \\
\hline \multirow[t]{4}{*}{19} & & 6 & 10 & 86 & & 103 & 21 & 146 & & 70 & 112 & 95 & 308 \\
\hline & 1,000 & 9 & 143 & 125 & 32.7 & 88 & 185 & 133 & 38 & 113 & 201 & 144 & 40.0 \\
\hline & 2,000 & 72 & 110 & 95 & 90.57 & 190 & 387 & 287 & 306. & 78 & 141 & 104 & 239 \\
\hline & 1,000 & 121 & 197 & 142 & 983.8203 & 35 & 156 & 80 & 708 & 24 & 67 & 45 & 322.8129 \\
\hline \multirow[t]{4}{*}{20} & 500 & 51 & 74 & 60 & 0.0468 & 55 & 114 & 73 & 0.0156 & 53 & 83 & 68 & 0.0312 \\
\hline & 1,000 & 54 & 78 & 62 & 0.031 & 55 & 117 & 73 & & 93 & 143 & 115 & 0.0468 \\
\hline & 2,000 & 44 & 74 & 58 & 0.031 & 58 & 130 & 87 & & 33 & 61 & 39 & 0.0156 \\
\hline & 1,000 & 43 & 69 & 57 & & 52 & 115 & 71 & & 42 & 75 & 59 & \\
\hline \multirow[t]{4}{*}{21} & & 4 & 9 & 8 & & & & & & 8 & 1 & & \\
\hline & 1,00 & 5 & 11 & 10 & & & & 1 & & 9 & & & \\
\hline & & 5 & 11 & 10 & & 10 & 20 & 19 & & 9 & 20 & 19 & 1.8252 \\
\hline & 1,000 & 5 & 11 & 10 & 6.63 & 11 & 23 & 22 & 15.3973 & 10 & 21 & 20 & 13.8997 \\
\hline \multirow[t]{4}{*}{22} & 500 & 50 & 91 & 68 & 1.1076 & 86 & 207 & 133 & 2.2308 & 36 & 92 & 65 & 1.0764 \\
\hline & 1,000 & 33 & 96 & 52 & & 52 & 146 & 100 & 6.552 & 43 & 104 & 71 & 4.602 \\
\hline & 2,000 & 27 & 113 & 48 & & 43 & 147 & 91 & 23.6186 & 52 & 167 & 83 & 22.9477 \\
\hline & 1,000 & 102 & 222 & 142 & 246.5128 & 76 & 196 & 140 & 244.2664 & 42 & 123 & 71 & 124.442 \\
\hline \multirow[t]{4}{*}{23} & 500 & 4 & 32 & 8 & 0.1092 & 13 & 69 & 30 & 0.4368 & 3 & 36 & 6 & 0.078 \\
\hline & 1,000 & 6 & 69 & 9 & & 17 & 230 & 61 & & 3 & 57 & 5 & 0.234 \\
\hline & & 5 & 61 & 8 & & 16 & & 41 & & 3 & 37 & 3 & 0.5304 \\
\hline & 1,000 & 5 & 62 & 11 & & 12 & 125 & 20 & 30.6074 & 3 & 56 & 5 & 6.1932 \\
\hline \multirow[t]{4}{*}{24} & 500 & 59 & 137 & 88 & 0.0156 & 62 & 232 & 133 & 0.0312 & 34 & 192 & 121 & 0.0156 \\
\hline & 1,000 & 26 & 91 & 34 & 0.0156 & 34 & 174 & 98 & 0.0312 & 56 & 292 & 204 & 0.0624 \\
\hline & 2,000 & 17 & 68 & 26 & 0.0156 & 16 & 112 & 35 & 0.0312 & 22 & 272 & 172 & 0.0624 \\
\hline & 1,000 & 37 & 89 & 42 & 0.1092 & 34 & 164 & 59 & 0.156 & 25 & 266 & 146 & 0.2496 \\
\hline
\end{tabular}


Table 2 (Continued)

\begin{tabular}{|c|c|c|c|c|c|c|c|c|c|c|c|c|c|}
\hline \multirow[t]{2}{*}{ No. } & \multirow[t]{2}{*}{ Dim } & \multicolumn{4}{|l|}{ LY } & \multicolumn{4}{|c|}{ NMLY2 } & \multicolumn{4}{|c|}{ NMLY1 } \\
\hline & & it & nf & ng & $t$ & it & nf & $\mathrm{ng}$ & $t$ & it & $n f$ & ng & $t$ \\
\hline \multirow[t]{4}{*}{25} & 500 & 7 & 67 & 43 & 12.8545 & 13 & 85 & 53 & 15.9589 & 13 & 80 & 26 & 10.4521 \\
\hline & 1,000 & 7 & 59 & 28 & 26.7698 & 8 & 78 & 51 & 41.9019 & 47 & 229 & 130 & 115.8931 \\
\hline & 2,000 & 22 & 141 & 90 & 275.5914 & 7 & 55 & 28 & 96.081 & 7 & 68 & 28 & 107.2507 \\
\hline & 1,000 & 7 & 63 & 36 & 716.4346 & 16 & 175 & 117 & 2148.9606 & 11 & 115 & 63 & 1265.6829 \\
\hline \multirow[t]{4}{*}{26} & 500 & 54 & 105 & 77 & 1.6224 & 55 & 127 & 88 & 1.7628 & 46 & 97 & 68 & 1.3728 \\
\hline & 1,000 & 53 & 101 & 75 & 5.4132 & 92 & 195 & 140 & 10.4053 & 46 & 94 & 69 & 5.0856 \\
\hline & 2,000 & 42 & 89 & 62 & 15.1945 & 71 & 162 & 113 & 27.5654 & 40 & 92 & 65 & 16.0213 \\
\hline & 1,000 & 29 & 68 & 43 & 44.4603 & 58 & 150 & 105 & 110.0275 & 44 & 97 & 68 & 69.7168 \\
\hline \multirow[t]{4}{*}{27} & 500 & 3 & 52 & 4 & 0.2184 & 11 & 178 & 91 & 1.9968 & 9 & 152 & 12 & 0.6708 \\
\hline & 1,000 & 13 & 193 & 99 & 8.7049 & 5 & 68 & 13 & 1.5756 & 7 & 106 & 27 & 2.9484 \\
\hline & 2,000 & 5 & 101 & 46 & 16.3645 & 7 & 141 & 40 & 16.5205 & 7 & 141 & 48 & 18.7045 \\
\hline & 1,000 & 9 & 112 & 32 & 99.279 & 8 & 140 & 33 & 112.5859 & 13 & 224 & 48 & 172.6151 \\
\hline \multirow[t]{4}{*}{28} & 500 & 8 & 23 & 10 & 0 & 22 & 60 & 38 & 0.0468 & 19 & 45 & 27 & 0.0156 \\
\hline & 1,000 & 9 & 25 & 13 & 0.0156 & 7 & 45 & 31 & 0.0156 & 11 & 59 & 43 & 0.0312 \\
\hline & 2,000 & 16 & 60 & 26 & 0.0312 & 13 & 78 & 42 & 0.0624 & 16 & 72 & 36 & 0.0468 \\
\hline & 1,000 & 5 & 22 & 7 & 0.0312 & 10 & 53 & 30 & 0.2496 & 11 & 60 & 40 & 0.2808 \\
\hline \multirow[t]{4}{*}{29} & 500 & 28 & 65 & 41 & 0.0468 & 13 & 69 & 52 & 0.0312 & 13 & 50 & 27 & 0.0312 \\
\hline & 1,000 & 4 & 17 & 5 & 0 & 22 & 86 & 53 & 0.0468 & 11 & 56 & 34 & 0.0156 \\
\hline & 2,000 & 7 & 23 & 8 & 0 & 10 & 49 & 26 & 0.0468 & 13 & 66 & 35 & 0.0468 \\
\hline & 1,000 & 7 & 26 & 9 & 0.0624 & 4 & 40 & 28 & 0.156 & 12 & 124 & 101 & 0.7332 \\
\hline \multirow[t]{4}{*}{30} & 500 & 287 & 452 & 387 & 14.6017 & 365 & 691 & 649 & 21.0913 & 249 & 380 & 334 & 10.1089 \\
\hline & 1,000 & 292 & 456 & 407 & 44.50 & 343 & 646 & 593 & 69.37 & 257 & 404 & 359 & 40.8411 \\
\hline & 2,000 & 343 & 524 & 477 & 154.3786 & 376 & 721 & 655 & 213.9242 & 271 & 457 & 376 & 122.1176 \\
\hline & 1,000 & 310 & 474 & 424 & 640.2749 & 376 & 729 & 663 & 1006.2533 & 236 & 412 & 337 & 512.9157 \\
\hline \multirow[t]{4}{*}{31} & 500 & 70 & 110 & 87 & 0.3276 & 73 & 158 & 113 & 0.39 & 58 & 115 & 90 & 0.2964 \\
\hline & 1,000 & 49 & 88 & 66 & 0.468 & 50 & 131 & 93 & 0.624 & 30 & 68 & 45 & 0.312 \\
\hline & 2,000 & 38 & 69 & 49 & 0.7644 & 64 & 142 & 103 & 1.3416 & 34 & 75 & 49 & 0.6396 \\
\hline & 1,000 & 27 & 52 & 32 & 2.0904 & 53 & 128 & 90 & 6.1152 & 34 & 85 & 60 & 4.0248 \\
\hline \multirow[t]{4}{*}{32} & 500 & 6 & 50 & 9 & 0.0156 & 53 & 144 & 89 & 0.0624 & 4 & 60 & 6 & 0 \\
\hline & 1,000 & 30 & 88 & 44 & 0.0468 & 18 & 211 & 35 & 0.046 & 6 & 93 & 7 & 0.0156 \\
\hline & 2,000 & 3 & 23 & 6 & 0.0156 & 10 & 107 & 16 & 0.0468 & 4 & 45 & 5 & 0.0156 \\
\hline & 1,000 & 5 & 39 & 10 & 0.0936 & 8 & 80 & 17 & 0.1872 & 4 & 55 & 8 & 0.0936 \\
\hline 33 & 500 & 320 & 442 & 406 & 7.6596 & 427 & 822 & 614 & 11.8405 & 4 & 24 & 7 & 0.1248 \\
\hline & 1,000 & 268 & 381 & 342 & 20.6233 & 338 & 652 & 483 & 29.1566 & 4 & 25 & 7 & 0.39 \\
\hline & 2,000 & 195 & 269 & 248 & 47.1435 & 235 & 461 & 342 & 66.7996 & 4 & 25 & 7 & 1.2792 \\
\hline & 1,000 & 145 & 198 & 182 & 137.4681 & 223 & 434 & 326 & 247.1524 & 4 & 25 & 7 & 4.5396 \\
\hline 34 & 500 & 105 & 187 & 146 & 0.1404 & 101 & 234 & 162 & 0.156 & 98 & 191 & 143 & 0.1404 \\
\hline & 1,000 & 33 & 76 & 49 & 0.1248 & 73 & 197 & 136 & 0.2496 & 97 & 203 & 156 & 0.2028 \\
\hline & 2,000 & 123 & 226 & 172 & & 173 & 416 & 306 & 0.6708 & 89 & 196 & 141 & 0.312 \\
\hline & 1,000 & 175 & 311 & 243 & 6676 & 236 & 537 & 395 & 4.61 & 151 & 319 & 239 & 2.6676 \\
\hline 35 & 500 & 12 & 25 & 14 & 0.0468 & 13 & 35 & 23 & 0.078 & 5 & 18 & 5 & 0.0156 \\
\hline & 1,000 & 12 & 25 & 14 & 0.078 & 11 & 30 & 19 & 0.1248 & 5 & 18 & 5 & 0.0312 \\
\hline & 2,000 & 11 & 23 & 13 & 0.1716 & 11 & 34 & 23 & 0.2808 & 5 & 18 & 5 & 0.0624 \\
\hline & 1,000 & 10 & 23 & 14 & 0.9984 & 10 & 31 & 22 & 1.56 & 5 & 18 & 5 & 0.2808 \\
\hline 36 & 500 & 37 & 228 & 122 & 0.6864 & 10 & 78 & 17 & 0.1404 & 9 & 34 & 16 & 0.078 \\
\hline & 1,000 & 17 & 75 & 25 & 0.2652 & 11 & 78 & 20 & 0.234 & 9 & 51 & 13 & 0.156 \\
\hline & 2,000 & 37 & 137 & 57 & 1.1076 & 11 & 101 & 21 & 0.5148 & 15 & 90 & 34 & 0.6864 \\
\hline & 1,000 & 16 & 82 & 23 & 2.496 & 10 & 82 & 18 & 2.1684 & 15 & 76 & 21 & 2.106 \\
\hline 37 & 500 & 11 & 25 & 16 & 0.0156 & 10 & 33 & 19 & 0 & 3 & 17 & 6 & 0 \\
\hline & 1,000 & 12 & 26 & 15 & 0.0156 & 11 & 29 & 19 & 0.0156 & 3 & 17 & 6 & 0 \\
\hline & 2,000 & 10 & 22 & 12 & 0.0312 & 10 & 27 & 18 & 0.0312 & 3 & 18 & 6 & 0.0156 \\
\hline & 5,000 & 8 & 18 & 10 & 0.0936 & 10 & 27 & 18 & 0.156 & 3 & 18 & 6 & 0.0624 \\
\hline 38 & 500 & 6 & 75 & 50 & 0.0156 & 10 & 123 & 72 & 0.0624 & 8 & 105 & 51 & 0.0312 \\
\hline & 1,000 & 4 & 63 & 22 & 0.0156 & 7 & 107 & 43 & 0.0468 & 4 & 56 & 24 & 0.0156 \\
\hline & 2,000 & 7 & 121 & 65 & 0.0624 & 13 & 211 & 130 & 0.1248 & 8 & 132 & 68 & 0.078 \\
\hline & 5,000 & 5 & 95 & 49 & 0.2184 & 5 & 84 & 34 & 0.156 & 6 & 115 & 51 & 0.2496 \\
\hline
\end{tabular}


Table 2 (Continued)

\begin{tabular}{|c|c|c|c|c|c|c|c|c|c|c|c|c|c|}
\hline \multirow[t]{2}{*}{ No. } & \multirow[t]{2}{*}{ Dim } & \multicolumn{4}{|l|}{ LY } & \multicolumn{4}{|c|}{ NMLY2 } & \multicolumn{4}{|c|}{ NMLY1 } \\
\hline & & it & $n f$ & $\mathrm{ng}$ & $t$ & it & nf & ng & $t$ & it & $n f$ & $\mathrm{ng}$ & $t$ \\
\hline \multirow[t]{4}{*}{39} & 500 & 8 & 127 & 51 & 2.7924 & 10 & 168 & 76 & 3.7596 & 9 & 127 & 62 & 3.042 \\
\hline & 1,000 & 7 & 107 & 40 & 8.5021 & 11 & 188 & 85 & 15.7405 & 9 & 144 & 61 & 11.9341 \\
\hline & 2,000 & 10 & 142 & 67 & 46.7691 & 16 & 248 & 104 & 81.2453 & 113 & 573 & 189 & 182.7708 \\
\hline & 5,000 & 9 & 165 & 72 & 698.6037 & 12 & 162 & 78 & 675.7495 & 13 & 181 & 83 & 756.8077 \\
\hline \multirow[t]{4}{*}{40} & 500 & 170 & 257 & 218 & 0.1092 & 305 & 578 & 430 & 0.1716 & 105 & 171 & 140 & 0.1404 \\
\hline & 1,000 & 153 & 231 & 196 & 0.0624 & 265 & 505 & 367 & 0.1404 & 114 & 208 & 155 & 0.0624 \\
\hline & 2,000 & 147 & 222 & 190 & 0.0936 & 272 & 522 & 390 & 0.1872 & 117 & 188 & 155 & 0.078 \\
\hline & 5,000 & 144 & 233 & 184 & 0.3588 & 305 & 579 & 425 & 0.8424 & 125 & 205 & 172 & 0.3276 \\
\hline
\end{tabular}

By $\left\|g_{k}\right\|^{2} \geq \beta\left(C_{k}-f\left(x^{*}\right)\right)$, we have

$$
C_{k+1}-f\left(x^{*}\right) \leq \vartheta\left(C_{k}-f\left(x^{*}\right)\right),
$$

where $\vartheta=1-c c_{2} \rho \beta\left(1-\zeta_{\max }\right) \in(0,1)$.

In the second case, $\left\|g_{k}\right\|^{2}<\beta\left(C_{k}-f\left(x^{*}\right)\right)$. By (19) and (30), we have

$$
f_{k+1}-f\left(x^{*}\right) \leq \tau\left(1+c_{1} v L\right)^{2}\left\|g_{k}\right\|^{2} \leq \tau \beta\left(1+c_{1} v L\right)^{2}\left(C_{k}-f\left(x^{*}\right)\right) .
$$

By combining the equality, the first equation of (32), and $Q_{k+1} \leq \frac{1}{1-\zeta_{\max }}, \zeta_{\max }<1$ and (31), we obtain

$$
\begin{aligned}
C_{k+1}-f\left(x^{*}\right) & \leq \frac{\zeta_{k} Q_{k}\left(C_{k}-f\left(x^{*}\right)\right)+\tau \beta\left(1+c_{1} \nu L\right)^{2}\left(C_{k}-f\left(x^{*}\right)\right)}{1+\zeta_{k} Q_{k}} \\
& =\left(1-\frac{1-\tau \beta\left(1+c_{1} v L\right)^{2}}{Q_{k+1}}\right)\left(C_{k}-f\left(x^{*}\right)\right) \\
& =\left(1-\left(1-\tau \beta\left(1+c_{1} v L\right)^{2}\right)\left(1-\zeta_{\max }\right)\right)\left(C_{k}-f\left(x^{*}\right)\right) \\
& =\left(1-c c_{2} \rho \beta\left(1-\zeta_{\max }\right)\right)\left(C_{k}-f\left(x^{*}\right)\right) \\
& \leq \vartheta\left(C_{k}-f\left(x^{*}\right)\right) .
\end{aligned}
$$

By (11), (33), and (34), we have

$$
f_{k}-f\left(x^{*}\right) \leq C_{k}-f\left(x^{*}\right) \leq \vartheta\left(C_{k-1}-f\left(x^{*}\right)\right) \leq \cdots \leq \vartheta^{k}\left(C_{0}-f\left(x^{*}\right)\right) .
$$

The proof is completed.

\section{Numerical experiments}

In this section, we report numerical results to illustrate the performance of hybrid conjugate gradient (LY) in [5], Algorithm 1 (NHLYCG1) and Algorithm 2 (NGLYCG2), in which (8) replaces only (9) in Step 2 of Algorithm 1. All codes are written with Matlab R2012a and are implemented on a PC with CPU $2.40 \mathrm{GHz}$ and 2.00GB RAM. We select 12 small-scale and 28 large-scale unconstrained optimization test functions from [15] and the CUTEr collection $[16,17]$ (see Table 1 ). All algorithms implement the stronger version of the Wolfe condition with $\rho=0.45$ and $\sigma=0.39$, and $\mu=0.5, \lambda=0.6, C_{0}=f_{0}, Q_{0}=1$, 


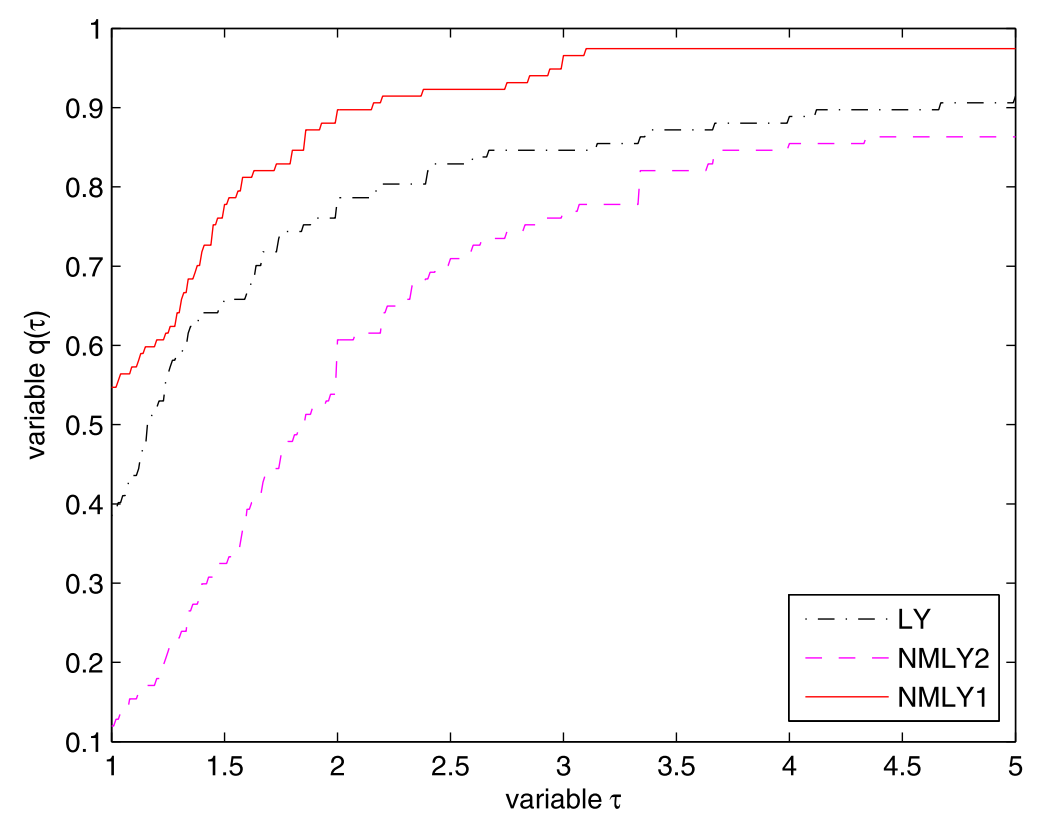

Figure 1 Performance profile comparing the number of iterations.

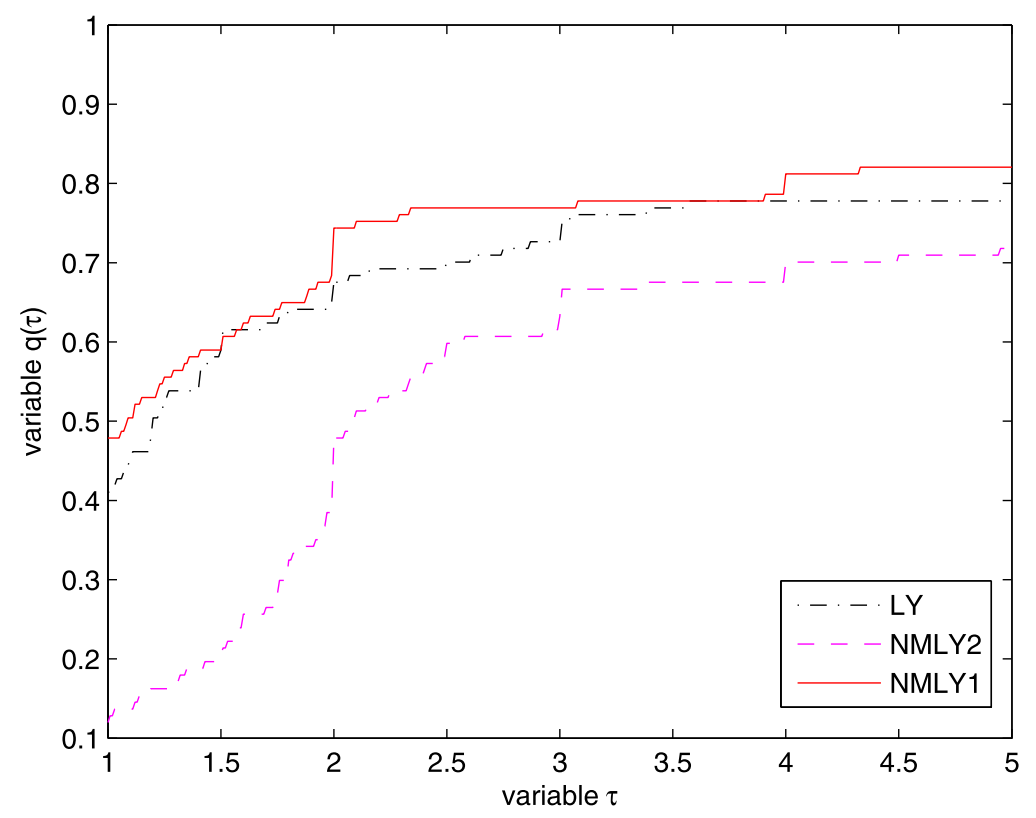

Figure 2 Performance profile comparing the CPU time.

$\zeta_{0}=0.08, \zeta_{1}=0.04, \zeta_{k+1}=\frac{\zeta_{k}+\zeta_{k-1}}{2}$, and the terminated condition

$$
\left\|g_{k}\right\|_{2} \leq 10^{-6} \text { or } \quad\left|f_{k+1}-f_{k}\right| \leq 10^{-6} \max \left\{1.0,\left|f_{k}\right|\right\}
$$

Table 2 lists all the numerical results, which include the order numbers and dimensions of the tested problems, the number of iterations (it), the function evaluations (nf), the 


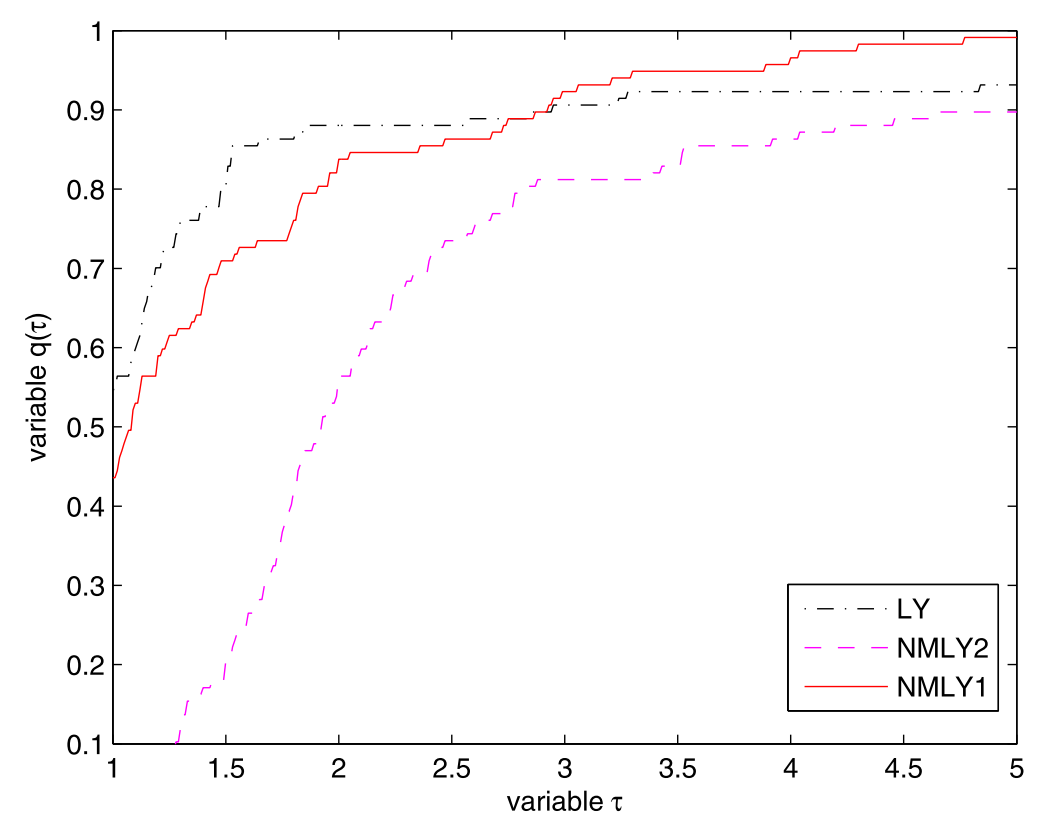

Figure 3 Performance profile comparing the number of function evaluations.

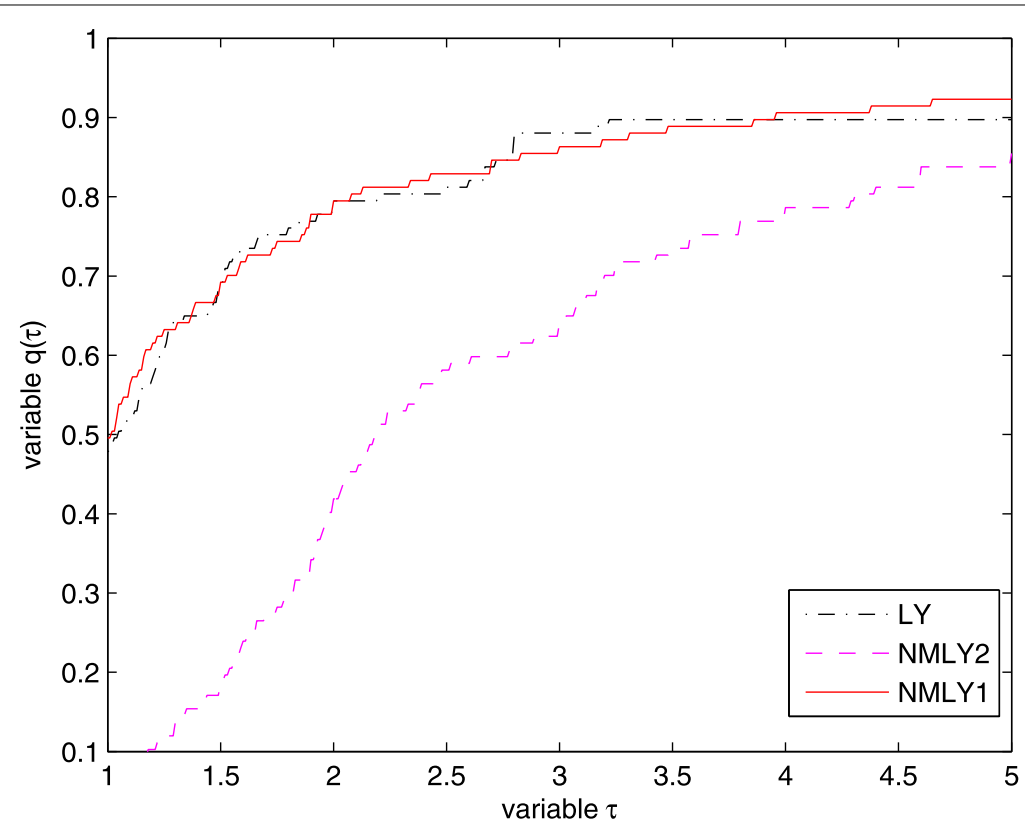

Figure 4 Performance profile comparing the number of gradient evaluations.

gradient evaluations (ng), and the CPU time ( $\mathrm{t}$ ) in seconds, respectively. We presented the Dolan and Moré [18] performance profiles for the LY, NHLYCG1, and NGLYCG2. Note that the performance ratio $q(\tau)$ is the probability for a solver $s$ for the tested problems with the factor $\tau$ of the smallest cost. As we can see from Figure 1 and Figure 2, NHLYCG1 is superior to LY and NGLYCG2 for the number of iterations and CPU time. Figure 3 shows that NGLYCG1 is slightly better than LY and NGLYCG2 for the number of function value 
evaluations. Figure 4 shows the performance of NGLYCG1 is very much like that of LY for the number of gradient evaluations. However, the performance of NGLYCG2 with the nonmonotone framework (3) is less than satisfactory.

\section{Competing interests}

The authors declare that they have no competing interests.

\section{Authors' contributions}

All authors contributed equally to the writing of this paper. All authors read and approved the final manuscript.

\section{Acknowledgements}

This work is supported in part by the NNSF (11171003) of China.

Received: 16 January 2015 Accepted: 27 March 2015 Published online: 08 April 2015

\section{References}

1. Hager, WW, Zhang, H: A new conjugate gradient method with guaranteed descent and an efficient line search. SIAM J. Optim. 16, 170-192 (2005)

2. Andrei, $\mathrm{N}$ : A hybrid conjugate gradient algorithm for unconstrained optimization as a convex combination of Hestenes-Stiefel and Dai-Yuan. Stud. Inform. Control 17(4), 55-70 (2008)

3. Babaie-Kafaki, S, Mahdavi-Amiri, N: Two modified hybrid conjugate gradient methods based on a hybrid secant equation. Math. Model. Anal. 18(1), 32-52 (2013)

4. Dai, YH, Yuan, Y: An efficient hybrid conjugate gradient method for unconstrained optimization. Ann. Oper. Res. 103, 33-47 (2001)

5. Lu, YL, Li, WY, Zhang, CM, Yang, YT: A class new conjugate hybrid gradient method for unconstrained optimization. J. Inf. Comput. Sci. 12(5), 1941-1949 (2015)

6. Yang, YT, Cao, MY: The global convergence of a new mixed conjugate gradient method for unconstrained optimization. J. Appl. Math. 2012, 93298 (2012)

7. Zheng, XF, Tian, ZY, Song, LW: The global convergence of a mixed conjugate gradient method with the Wolfe line search. Oper. Res. Trans. 13(2), 18-24 (2009)

8. Touati-Ahmed, D, Storey, C: Efficient hybrid conjugate gradient techniques. J. Optim. Theory Appl. 64(2), 379-397 (1990)

9. Grippo, L, Lampariello, F, Lucidi, S: A nonmonotone line search technique for Newton's method. SIAM J. Numer. Anal. 23, 707-716 (1986)

10. Zhang, $\mathrm{H}$, Hager, WW: A nonmonotone line search technique and its application to unconstrained optimization. SIAM J. Optim. 14, 1043-1056 (2004)

11. Zoutendijk, G: Nonlinear programming, computational methods. In: Abadie, J (ed.) Integer and Nonlinear Programming. North-Holland, Amsterdam (1970)

12. Al-Baali, M: Descent property and global convergence of the Fletcher-Reeves method with inexact line search. IMA J. Numer. Anal. 5, 121-124 (1985)

13. Gilbert, JC, Nocedal, J: Global convergence properties of conjugate gradient methods for optimization. SIAM J. Optim. 2(1), 21-42 (1992)

14. Yu, GH, Zhao, YL, Wei, ZX: A descent nonlinear conjugate gradient method for large-scale unconstrained optimization. Appl. Math. Comput. 187(2), 636-643 (2007)

15. Moré, JJ, Garbow, BS, Hillstrom, KE: Testing unconstrained optimization software. ACM Trans. Math. Softw. 7, 17-41 (1981)

16. Andrei, N: An unconstrained optimization test functions collection. Adv. Model. Optim. 10, 147-161 (2008)

17. Gould, NIM, Orban, D, Toint, PL: CUTEr and SifDec: a constrained and unconstrained testing environment, revisited. ACM Trans. Math. Softw. 29, 373-394 (2003)

18. Dolan, ED, Moré, JJ: Benchmarking optimization software with performance profiles. Math. Program. 9, 201-213 (2002) 